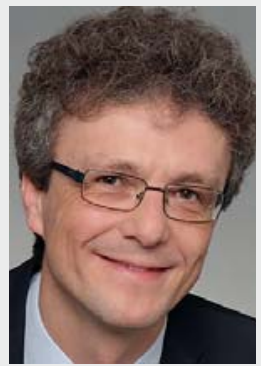

Axel Hübler

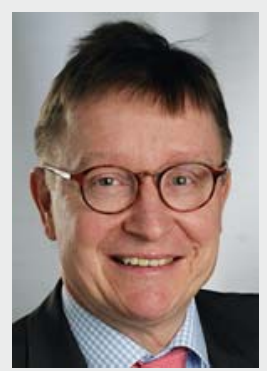

Roland Hentschel

\title{
Ärztinnen und Wissenschaftlerinnen in der Neonatologie
}

\author{
Liebe Leserinnen, liebe Leser,
}

diese Ausgabe der Neonatologie Scan erscheint pünktlich zum Weltfrauentag, welcher 1911 als Initiative für Gleichberechtigung, das Wahlrecht für Frauen sowie die Emanzipation von Arbeiterinnen entstand und seit 1921 am 8. März stattfindet. Wir sind in diesem Zusammenhang sehr dankbar, dass diesmal ausschließlich Ärztinnen und Journalistinnen, welche sich in unserem Fachgebiet engagieren, den Diskussionsteil, die Weiterbildungsartikel und die Studienzusammenfassungen gestaltet haben. Eine Schlüsselrolle für das Gelingen der Neonatologie Scan nehmen seit geraumer Zeit bei jeder Ausgabe wieder neu Frau Olga Münch (Thieme-Verlag) und Frau Beate Neth (Firma „seitenweise“) ein. Wir möchten Frau Münch und Frau Neth, die stets verlässlich für uns alle im Hintergrund arbeiten, an dieser Stelle für die exzellente Zusammenarbeit herzlichst „Danke“ sagen.

Das einhundertste Jubiläum des Weltfrauentages ist für uns auch Anlass, den Stand der Geschlechtergerechtigkeit und die historischen Leistungen von Ärztinnen und Wissenschaftlerinnen in unserem Fachgebiet anhand einiger Beispiele zu sichten.

Wussten Sie, dass die 1977 gegründete Gesellschaft für Neonatologie und Pädiatrische Intensivmedizin (GNPI) nach zehn männlichen Präsidenten erstmals seit 2017 mit Ursula Felderhoff-Müser von einer Frau geleitet wird? Neonatologen organisierten im deutschsprachigen Raum seit 1970 bisher siebenundvierzig Symposien und Jahrestagungen, wobei bisher zweimal Frauen federführend beteiligt waren. Der Deutschen Gesellschaft für Kinder- und Jugendmedizin e. V. (DGKJ), 1883 gegründet, folgte nach fünfundsechzig Männern an der Spitze mit Ingeborg KrägelohMann 2018 erstmals eine Frau als Präsidentin, 2019 auch als erste Kongresspräsidentin einer Jahrestagung der DGKJ. Trotz dieses strukturellen Ungleichgewichts in fachpolitischen Leitungsfunktionen zuungunsten von Frauen wäre die Entwicklung der Pädiatrie und insbesondere der Neonatologie in den letzten mehr als einhundert Jahren ohne eine Vielzahl herausragender klinischer und wissenschaftlicher Leistungen von Ärztinnen, Kinderkrankenschwestern, Hebammen und Vertreterinnen weiterer Disziplinen nicht denkbar gewesen.

Beginnend ab den 1870er Jahren wendeten sich Ärztinnen der Frauen- und Kindermedizin zu. Ursächlich dafür war allerdings zunächst vor allem der Umstand, dass ihnen die Beschäftigung mit der Chirurgie als klassischer Männerdomäne verwehrt wurde. Entscheidende Impulse gingen in dieser Zeit auch eher weniger von den Wissenschaften aus, sondern von sozialen und politischen Aktivitäten wie jener der Infant Welfare Movement, welche zur Gesundheitserziehung der Mütter, zur Förderung des Stillens und zur Beschäftigung mit der Kindersterblichkeit beitrugen. Ein Medizinstudium war Frauen im 19. Jahrhundert in einigen europäischen Ländern wie Frankreich und der Schweiz sowie in Amerika möglich. An preußischen Universitäten wurden Frauen jedoch erst ab dem Jahre 1908 als Studierende und ab 1914 zur Approbation zugelassen. So wurde beispielsweise Emilie Lehmus (1841-1932), nachdem sie in Zürich ein Medizinstudium mit dem Prädikat ausgezeichnet absolvierte, in Deutschland zunächst die Anerkennung der Arztausbildung verweigert. In Berlin, wo sie sich niederließ, gründete sie gemeinsam mit Franziska Tiburtius (1843-1927) eine „Poliklinik weiblicher Ärzte für Frauen und Kinder“ und 1881 eine „Pflegeanstalt für Frauen“, die nachfolgend zu einer modernen chirurgischen 
Klinik entwickelt wurde und insbesondere jungen Ärztinnen Weiterbildungsmöglichkeiten bot. Beide Medizinerinnen engagierten sich für die Frauenrechte. Emilie Lehmus unterstützte die Vereinigung weiblicher Ärzte, welche 1908 gegründet wurde, mit 16000 Reichsmark, und Franziska Tiburtius setzte sich insbesondere für die Aufhebung des Studierverbots für Frauen in Deutschland ein. In Frankreich hatte zu dieser Zeit Félicie Henry, von 1881 bis 1895 leitende Hebamme im Hôpital de la Charité in Paris, gemeinsam mit Stéphane Étienne Tarnier (1828-1897) entscheidenden Anteil an der Einrichtung einer ersten Station für Frühgeborene und der Entwicklung eines Inkubators („couveuse“). Nach Etablierung der neuen Strukturen, in deren Folge die Überlebensraten auf seinerzeit bemerkenswerte $51 \%$ stiegen, untersagte jedoch der Klinikleiter Pierre-Constant Budin (1846-1907) weiblichen Medizinerinnen alle Interventionen an den kleinen Patienten außer der Gabe von Sauerstoff. Genauere Lebensdaten sind von Félicie Henry, im Gegensatz zu ihren männlichen ärztlichen Kollegen, in der typischen Primärliteratur nicht verzeichnet [1-7].

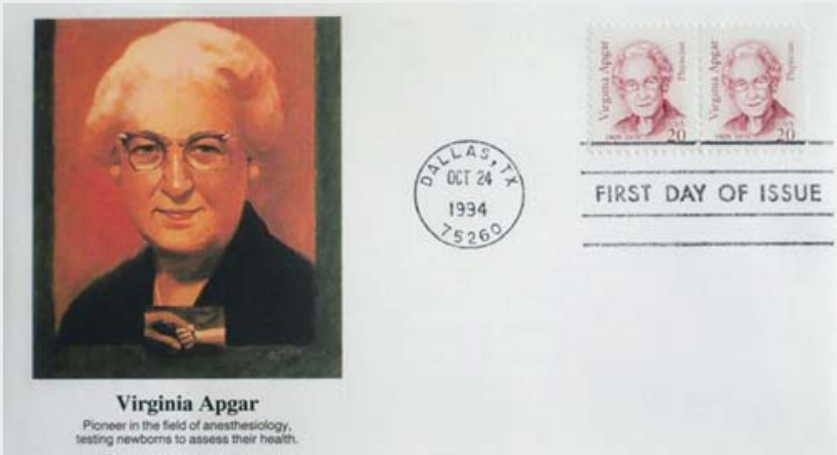

Anlässlich ihres 20. Todestages wurde Virginia Apgar von der US-Post mit einer Porträtbriefmarke in der Serie „Great Americans“ geehrt. Die Erstausgabe der Apgar-Briefmarke auf einem sog. Ganzsachenbrief zeigt Virginia Apgar im Alter von etwa 60 Jahren. (Bild: Sammlung M. David). Quelle: David M, Ebert AD. Berühmte Ärzte. Virginia Apgar (1909-1974) und der Apgar-Score. Geburtshilfe und Frauenheilkunde 2014; 74 (11): 992-994. doi:10.1055-/s-0034-1383255
Am Columbia University College of Physicians and Surgeons in New York legte 1933 Virginia Apgar (1909-1974) als eine der ersten Frauen ihr Examen ab und startete danach eine sehr erfolgreiche Ausbildung als Chirurgin am Presbyterian Hospital in New York. Ihr Mentor Allen Whipple riet ihr trotz großer Begabung von der Chirurgie ab, da ihre Erfolgsaussichten als Frau schlecht waren. Im jungen Fachgebiet Anästhesie wurde sie 1938 die erste Leiterin der Anästhesieabteilung der Columbia University. Als die von ihr erfolgreich entwickelte und geleitete Abteilung elf Jahre später in ein eigenständiges Department umgewandelt wurde, erhielt die Stelle allerdings ein männlicher Kollege. Die Idee zu ihrem 1953 veröffentlichten Newborn Scoring System soll ihr bei einer Kaffeepause gekommen sein, während der sie ein Student fragte, nach welchen Kriterien sie ein Neugeborenes nach der Geburt einschätzen würde. Virginia Apgar war eine ausgezeichnete Violinistin, und ähnlich wie bei einer spontanen musikalischen Darbietung improvisierte sie eine rasche Antwort mit den wesentlichen klinischen Merkmalen. Überrascht von ihrer Idee, testete sie unverzüglich die fünf Zeichen (heart rate, respiratory rate, reflex irritability, muscle tone, color) im Kreißsaal in Bezug auf das postnatale Alter. Ihre darauf hin initiierte erste Studie enthielt die Informationen über 1760 Neugeborene. Mit Anwendung des Apgar Scores konnten nun jene Neugeborene identifiziert werden, welche einer Überwachung und einer Intervention bedurften, was die Prognose der betroffenen Patienten entscheidend verbesserte. Virginia Apgar hat damit eine bis heute gültige universelle Sprache für alle geschaffen, welche an der Versorgung Neugeborener beteiligt sind [8-10].

Eigentlich wollte Mary Ellen Avery (1927-2011) gleich nach dem Schulabschluss Medizin an der Harvard Medical School studieren. Aber diese nahm Frauen erst ab 1949 auf. So schloss sie zunächst Chemie 1948 am Wheaton College und Medizin 1952 an der John Hopkins University ab, absolvierte an letzterer auch ihre Facharztausbildung und ging danach mit einem Forschungsstipendium nach Harvard. Ihre eigene Tuberkulosekrankheit weckte möglicherweise das Interesse an Funktionen der Lunge. 1957 entdeckte sie in Harvard, dass das seinerzeit hyaline membrane disease genannte Atemnotsyndrom des Frühgeborenen durch einen Mangel an einer Substanz verursacht wird, welche die Oberflächenspannung der Alveolen reduziert. Ihr Konzept des Surfactant-Mangels wurde jedoch von anderen Wissenschaftlern zunächst angezweifelt, welche weiterhin Veränderungen der Lungen- 
zirkulation oder Fibrindefekte für die Erkrankung als ursächlich ansahen. Erst mehr als zwanzig Jahre später erlangten Mary Ellen Averys Forschungsergebnisse Bestätigung, als Tetsuro Fujiwara in Japan mit bovinem Surfactant erfolgreich Frühgeborene therapierte. Die Gabe von Surfactant wurde dann Anfang der 1980er Jahre als kausale Standardbehandlung des Atemnotsyndroms etabliert. Anhand einer ersten follow-up-Untersuchung konnte bereits 1986 gezeigt werden, dass die Therapie nicht mit ungünstigen Langzeitfolgen assoziiert war. Mit mehr als dreißig randomisierten Studien bei über 6000 Neugeborenen ist Surfactant bisher das meistuntersuchte Medikament in der Neonatologie und verbesserte die Überlebensraten von Frühgeborenen wesentlich [11-16].

Genau fünfzig Jahre nachdem an preußischen Universitäten erstmals Frauen nach dem Medizinstudium zur Approbation zugelassen wurden, erhielt 1964 Leonore Ballowitz (1923-1994) an der Medizinischen Fakultät der Freien Universität Berlin den Ruf als erste Professorin. Leonore Ballowitz studierte Medizin in Breslau, Freiburg und Berlin. An ihrem ersten Arbeitstag als Ärztin, dem 01. Juli 1948, wurde sie im Berliner Kaiserin-Auguste-Victoria Haus (KAVH) mit der Durchführung einer seinerzeit achtstündigen Blutaustauschtransfusion bei einem Neugeborenen betraut. Dies war wohl auch der erste Kontakt Leonore Ballowitz' mit ihrem hauptsächlichen späteren Forschungsgebiet, der Hyperbilirubinämie in Zusammenhang mit der Blutgruppenserologie, Leberenzymaktivitäten, Verdrängung des Bilirubins durch Medikamente aus der Proteinbindung und Effekten der Fototherapie. Leonore Ballowitz gehörte zu den Gründungsmitgliedern der Gesellschaft für Perinatale Medizin, war Herausgeberin der Zeitschrift Pädiatrische Praxis und bildete eine Vielzahl von Ärztinnen und Ärzten in ihrem Spezialgebiet aus. Nach ihrer Pensionierung 1984 widmete sie sich medizingeschichtlichen Themen, insbesondere der Aufarbeitung der Geschichte „ihres“ KAVH. Leonore Ballowitz wurde 1985 erstes Ehrenmitglied der GNPI, und bis zur Ernennung von Evelyn Kattner im Jahr 2015 blieb sie auch die einzige Frau, der diese Wertschätzung zu Teil wurde [17].

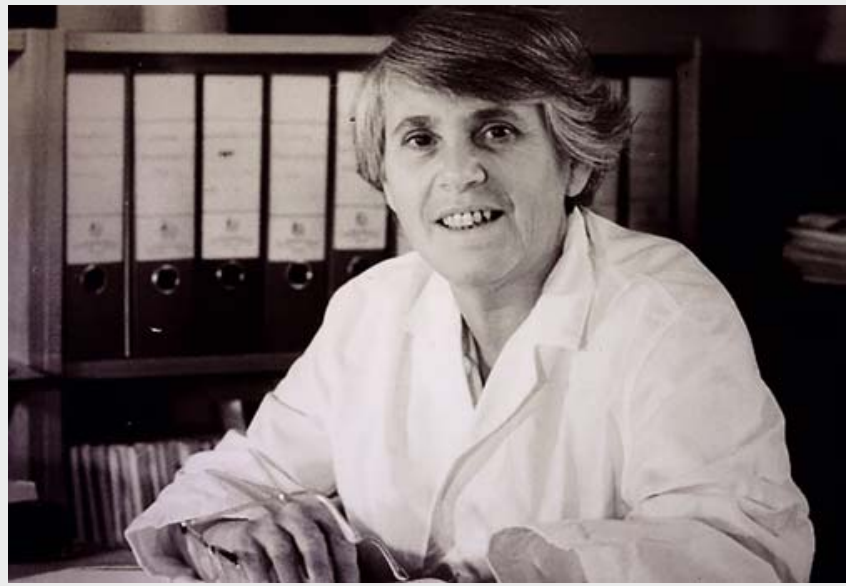

Frau Professor Dr. Ingeborg Syllm-Rapoport 1975 an ihrem Schreibtisch in der Berliner Charité (mit freundlicher Genehmigung von Herrn Professor Dr. Dieter Gmyrek, Radebeul).
Auf dem Gebiet der ehemaligen Deutschen Demokratischen Republik (DDR) geht die Etablierung einer modernen Neonatologie auf Ingeborg Syllm-Rapoport (1912-2017) zurück. Ingeborg Syllm-Rapoport wurde im heutigen Kamerun geboren, schloss 1937 in Hamburg das Medizinstudium ab und begann am dortigen Israelitischen Krankenhaus als Assistenzärztin. Aufgrund ihrer jüdischen Großeltern mütterlicherseits galt sie als Mischling ersten Grades und emigrierte im September 1938 in die USA. Dort holte Ingeborg Syllm-Rapoport am Women's Medical College ihr Examen nach und spezialisierte sich in verschiedenen Kliniken in der Pädiatrie. Gemeinsam mit ihrem Mann Samuel Mitja Rapoport, beide waren Mitglied der Kommunistischen Partei der USA, verließ sie 1950 im Zuge von Ermittlungen des House Committee on Un-American Activities gegen sie die USA. Das Ehepaar blieb zunächst in Österreich und wechselte 1952 in die DDR. Am Hufeland-Krankenhaus Berlin-Buch erhielt Ingeborg Syllm-Rapoport 1953 ihre Facharztanerkennung in der Kinderheilkunde und habilitierte sich anschließend am Institut für Biochemie der HumboldtUniversität Berlin. Ab 1959 war sie an der Kinderklinik der Charité tätig. 1969 wurde Ingeborg Syllm-Rapoport an der Humboldt-Universität Berlin auf den ersten europäischen Lehrstuhl für Neonatologie berufen, welchen sie bis zu ihrer Emeritierung 1973 innehatte. In den Fokus der Laienöffentlichkeit gelangte Ingeborg SyllmRapoport im Mai 2015, als die Universität Hamburg ihr als Geste der Wiedergut- 
machung des Unrechts aus der Nazizeit die prüfungslose Anerkennung eines Doctor honoris causa anbot. Sie lehnte dies ab und unterzog sich einer ordentlichen Prüfung, welche die Geschichte der Diphtherieforschung beinhaltete. Der historische Verdienst von Ingeborg Syllm-Rapoport bezieht sich wesentlich darauf, dass sie als Begründerin der modernen Neonatologie im deutschsprachigen Raum gelten kann. Große Bedeutung für die Entwicklung der Neonatologie kam dabei neben dem Aufbau einer ersten eigenständigen Abteilung an der Charité Ende der 1960er Jahre der Etablierung eines Forschungsprojektes „Perinatologie“ zu, in dem praktisch alle ostdeutschen Universitätsklinika vernetzt waren. Ergebnis der damit einhergehenden, ortsübergreifenden Zusammenarbeit war, dass sie neben ihren unmittelbaren Schülern und Mitstreitern Ludwig Grauel (1935-2005), Roland Wauer (1942-2020) und Dieter Gmyrek ( $\left.{ }^{*} 1933\right)$ auf dem Gebiet der ehemaligen DDR eine Generation von Neonatologen ausbildete, welche später selbst teilweise über die Landesgrenzen hinaus das Fachgebiet prägten [18-20].

In den USA begann in den 1970ern die aus Deutschland stammende Psychologin Heidelise Als systematisch das Verhalten Frühgeborener auf der neonatologischen Intensivstation zu beobachten. Aus den Überlegungen zu kindlichen Bedürfnissen, dem Einfluss der Umgebungsfaktoren und Fragen der Eltern-Kind-Interaktion entwarf Heidelise Als an der Harvard University Mitte der 1980er ihr Konzept Newborn Individualized Developmental Care and Assessment Program (NIDCAP), was Grundlagen legte, die physiologischen Besonderheiten dieser vulnerablen Patientengruppe besser zu berücksichtigen und nachfolgend mit zur Implementierung entwicklungsfördernder Ansätze in die neonatologische Intensivtherapie führte [21,22].

Ein Paradigmenwechsel im Management des Apnoe-Bradykardie-Hypoxämie-Syndroms (ABHS) wurde Mitte der 2000er Jahre durch die Arbeiten der ebenfalls aus Deutschland stammenden Barbara Schmidt, seinerzeit Hamilton (Kanada), später Philadelphia (USA), eingeleitet. Barbara Schmidt zeigte als Leiterin der Studie „Caffeine for Apnea of Prematurity“, welche über zweitausend Frühgeborene einschloss und erstmals 2006 publiziert wurde, dass der frühzeitige Einsatz des Medikamentes nicht nur effektiv die akuten Symptome, sondern auch das schadensfreie Langzeitüberleben in dieser vulnerablen Patientengruppe verbessert. Damit erhielten Neonatologen nach mehr als dreißig Jahren empirischer Behandlungsversuche der Apnoen mit unterschiedlichen Substanzen erstmals eine klare Datenbasis für die Therapiesteuerung, was nachfolgend den abgestuften und zeitigeren Einsatz weniginvasiver Beatmungsformen ermöglichte [23, 24].

Aus sorgfältiger klinischer Beobachtung und gezieltem, abwägendem Einsatz der Intensivmedizin heraus entwickelte Angela Kribs in Köln ab 2001 die Technik der wenig-invasiven Surfactant-Therapie. Am Anfang stand, so ihre persönliche Mitteilung, ein kleines Frühgeborenes der 23. Schwangerschaftswoche, welches aufgrund der schlechten Prognose palliativ versorgt werden sollte. Überraschenderweise atmete der kleine Knabe regelmäßig, war aber nicht zu oxygenieren. Da eine Intubation und maschinelle Beatmung mit den Eltern nicht vereinbart waren, entstand bei vermutetem schwerem Atemnotsyndrom aus der Akutsituation heraus bei Angela Kribs die Idee, das Surfactant unter Blähmanöver mit einer dünnen Magensonde zu applizieren. Der Vater stimmte dem individuellen Heilversuch zu, und das Kind stabilisierte sich daraufhin so gut, dass die anwesenden Kinderkrankenschwestern bei nachfolgenden Patienten meinten: „Frau Doktor, machen sie es doch wie damals... “. Mit Unterstützung durch Bernhard Roth wurde daraufhin in Köln eine Beobachtungsstudie initiiert, und die ermutigenden Ergebnisse erstmals 2003 zur Jahrestagung der GNPI, ebenfalls in Köln, vorgestellt. Wissenschaftliche Akzeptanz fand die Methode der early administration of surfactant in spontaneous breathing with nCPAP erst mit Publikation in Pediatric Anesthesia 2007. Danach folgten multizentrische Studien und die Implementierung und Verbreitung der wenig-invasiven Surfactanttherapie in die klinische Routine [25-27]. 
An dieser Stelle darf nicht unerwähnt bleiben, dass eine Vielzahl weiterer Frauen als Neonatologinnen in Deutschland in der Position einer Abteilungsleiterin und anderen verantwortungsvollen Funktionen hervorragende klinische und wissenschaftliche Arbeit geleistet haben bzw. leisten. Nicht wenige dieser Kolleginnen haben darüber hinaus durch aktive Beiträge alle bisherigen Ausgaben der Neonatologie Scan inhaltlich außerordentlich bereichert.

Mit Fragen der Geschlechtergerechtigkeit in Bezug auf Entwicklungsaussichten von Ärztinnen in der Neonatologie beschäftigte sich eine Ende 2020 im Journal of Perinatology publizierte anonyme Befragung unter 3575 Mitgliedern der American Academy of Pediatrics, Section Neonatal-Perinatal Medicine, auf welche 560 Antworten eingingen. Es zeigte sich nach Adjustierung an mögliche Kofaktoren, dass Neonatologinnen auch heute, die Studie bezieht sich konkret auf die Situation in den USA, signifikant seltener Führungspositionen innehaben, ein geringeres Gehalt beziehen und weniger Publikationen vorzuweisen können. Der Schlussfolgerung der Autoren der Arbeit „Equity for women in medicine - neonatologists identify issues“ schließen wir uns vorbehaltlos an: „Further action needs to be taken to inform action to reduce bias, champion equity, inform policy, and promote advocacy. “ [28]

Mit herzlichen Grüßen an Sie alle und den besten Wünschen an alle Kolleginnen zum Weltfrauentag verbleiben

Ihre Herausgeber

PD Dr. med. Axel Hübler

Chefarzt der Klinik für Kinder- und Jugendmedizin

Klinikum Chemnitz gGmbH

Prof. Dr. med. Roland Hentschel

Leiter des Funktionsbereichs Neonatologie/Intensivmedizin

Universitätsklinikum Freiburg

Literatur

[1] McCleary GF. The Early History of the Infant Welfare Movement. H:K. Lewis \& Company; 1933: 176

[2] „Emilie Lehmus“. Wikipedia, Die freie Enzyklopädie. Bearbeitungsstand: 23. September 2020, 20:06 UTC. https://de. wikipedia.org/w/index.php?title=Emilie_Lehmus\&oldid= 203922387 (Stand: 2. Februar 2021)

[3] „Franziska Tiburtius“. Wikipedia, Die freie Enzyklopädie. Bearbeitungsstand: 12. Oktober 2020, 00:57 UTC. https://de.wikipedia.org/w/index.php?title=Franziska_Tiburtius\&oldid $=204465653$ (Stand: 2 . Februar 2021)

[4] Bornemann R (Hrsg.) Erste weibliche Ärzte. Die Beispiele der „Fräulein Doctores“ Emilie Lehmus (1841-1932) und Franziska Tiburtius (1843-1927) - Biographisches und Autobiographisches. In: Brinkschulte E. Weibliche Ärzte. Die Durchsetzung eines Berufsbildes in Deutschland. Berlin: Edition Hentrich; 1993: 24-32

[5] SK: Vergessene Biographien (31). In: Verein zur Begleitung öffentlicher Diskussion in den Innenstadtbezirken e. V. (Hrsg.) Scheinschlag; 2004: http://www.scheinschlag.de/archiv/ 2004/05_2004/texte/17.html

[6] Gudrun Wedel. Autobiographien von Frauen: ein Lexikon. Böhlau Verlag; 2010: ISBN 3412205850

[7] Toubas PL, Nelson R. The Role of the French Midwives in Establishing the First Special Care Units for Sick Newborns. Journal of Perinatology 2020; 22: 75-77

[8] Apgar V. A proposal for a new method of evaluation of the newborn infant. Curr Res Anaesth Analg 1953; 32: 261-267. doi: 10.1213/00000539-195301000-00041

[9] „Virginia Apgar“. Wikipedia, Die freie Enzyklopädie. Bearbeitungsstand: 22. August 2020, 20:48 UTC. https://de.wikipedia.org/w/index.php?title=Virginia_Apgar\&oldid=203009678 (Stand: 2. Februar 2021) 
[10] Paul-Louis Toubas. Pioneer Women in Neonatology: Part 2: 1945-1960. NeoReviews 2011; 12: e303-e307. doi: 10.1542/neo.12-6-e303

[11] Paul-Louis Toubas. Pioneer Women in Neonatology: Part 3: The Roaring Sixties (1960-1980). NeoReviews 2011; 12: e361-e367. doi: 10.1542/neo.12-7-e361

[12] Avery ME, Mead J. Surface properties in relation to atelectasis and hyaline membrane disease. Am J Dis Child 1959; 97: 517-527

[13] Fujiwara T et al. Artificial surfactant therapy in hyaline-membrane disease. Lancet 1980; 1 : 55-59

[14] Hallman $M$ et al. Exogenous human surfactant for treatment of severe respiratory distress syndrome: a randomized prospective clinical trial. J Pediatr 1985; 106: 963-969

[15] Halliday HL, McClure G, Reid MM. Growth and development two years after artificial surfactant replacement at birth. Early Hum Dev 1986; 13: 323-327

[16] Cooke RW. Trends in preterm survival and incidence of cerebral haemorrhage 1980-9. Arch Dis Child 1991; 66: 403-407

[17] Lea Münch. Kinder und Kinderheilkunde in Berlin - Zwischen Fürsorge und Forschung (19451965). Dissertation. Berlin: 2020

[18] Ingeborg Rapoport. Meine ersten drei Leben: Erinnerungen. Nora; 2020: ISBN-10: 3935445814

[19] Wauer Roland R. Inge Rapoport - Nestorin der deutschen Neonatologie. Sitzungsberichte der Leibniz-Sozietät der Wissenschaften zu Berlin 2013; 115: 37-59

[20] „Ingeborg Rapoport“. Wikipedia, Die freie Enzyklopädie. Bearbeitungsstand: 29. Januar 2021, 15:07 UTC. https://de.wikipedia.org/w/index.php?title=Ingeborg_Rapoport\&oldid= 208208326 (Stand: 2. Februar 2021)

[21] Als H. NIDCAP: testing the effectiveness of a relationship-based comprehensive intervention. Pediatrics 2009; 124: 1208-1210

[22] Als $\mathrm{H}$ et al. Individualized developmental care for the very low-birth-weight preterm infant. Medical and neurofunctional effects. JAMA 1994; 272: 853-858

[23] Schmidt Barbara, Roberts Robin S, Davis Peter et al. for the Caffeine for Apnea of Prematurity Trial Group*. Caffeine Therapy for Apnea of Prematurity. N Engl J Med 2006; 354: 2112-2121. doi: 10.1056/NEJMoa054065

[24] https://www.med.upenn.edu/apps/faculty/index.php/g275/p8168945

[25] Angela Kribs, persönliche Information im Januar 2021.

[26] Kribs A, Pillekamp F, Hünseler C et al. Early administration of surfactant in spontaneous breathing with nCPAP: feasibility and outcome in extremely premature infants (postmenstrual age $\leq 27$ weeks). Paediatr Anaesth 2007; 17: 364-369. doi: 10.1111/j.1460-9592. 2006.02126.x

[27] Kribs A, Roll C, Göpel W et al. PMID: 26053341 Nonintubated Surfactant Application vs Conventional Therapy in Extremely Preterm Infants: A Randomized Clinical Trial. JAMA Pediatr 2015; 169: 723-730. doi: 10.1001/jamapediatrics.2015.0504

[28] Horowitz E, Randis TM, Samnaliev M et al. Equity for women in medicine - neonatologists identify issues. Journal of perinatology 2020: doi: 10.1038/s41372-020-00897-4 